\title{
Global VOR gain adaptation during near fixation to foveal targets
}

\author{
Jason A. Williams ${ }^{\mathrm{a}, *}$, Bruce Bridgeman ${ }^{\mathrm{b}}$, Tadg Woods ${ }^{\mathrm{c}}$, \\ Robert Welch ${ }^{\mathrm{d}}$ \\ a Department of Psychology, Gonzaga University, 501 E Boone Ave, AD54, Spokane, WA, United States \\ ${ }^{\mathrm{b}}$ Departments of Psychology and Psychobiology, University of California, Santa Cruz Social Sciences 1, \\ Santa Cruz, CA 95064, United States \\ c Department of Mathematics, Gonzaga University, 501 E Boone Ave, HC308A, Spokane, WA, United States \\ ${ }^{\mathrm{d}}$ NASA Ames-Research Center, Moffet Field, CA, United States
}

\begin{abstract}
Long-term rotational vestibulo-ocular (VOR) adaptation occurs during systematic dysmetria between visual and vestibular afferents, adjusting eye-rotation angular velocity to re-establish retinal stability of the visual field. Due to translational motion of the eyes during head rotation, VOR gain is higher when fixating near objects. The current study measures VOR in humans before and after $6 \mathrm{~min}$ of exposure to a foveal near-target during sinusoidal whole-body rotation at $0.45 \mathrm{~Hz}$. All of six participants showed post-exposure increases in open-loop VOR gain after fixating near targets, demonstrating a mean modulation increase of open-loop VOR gain from 0.86 before adaptation to 1.2 after adaptation. We discuss a number of theoretical and applied implications.
\end{abstract}

In many species, the rotational vestibulo-ocular reflex (VOR) efficiently stabilizes the visual field during head rotation by rotating the eyes in the direction opposite head movement based on semi-circular canal efferents; a complementary system, the linear vestibulo- 
ocular reflex (1-VOR) employs acceleration signals from the otilith organs to rotate the eyes during translational movements. Smooth pursuit and optokinetic systems also stabilize the retinal image. In order to operate properly, these systems must act in conjunction, a property particularly important for the two VOR systems, as combined linear and rotational head movements are common, e.g., during locomotion (Grossman, Leigh, Bruce, Huebner, \& Lanska, 1989). VOR must also interact with other oculomotor systems e.g., head movements often occur when tracking objects (Lisberger, 1990) or looking to new locations (Guitton \& Volle, 1987). Additionally, the VOR itself must be plastic because its gain (eye rotation/head rotation) must increase to stabilize near targets, and adapt over the long term to maturational size changes, alterations or damage to the nerves or semi-circular canals, and/or the introduction of optical distortion. While considerable research has examined the interaction of the two vestibular systems, vestibular interactions with the visually-driven oculomotor systems, and long-term VOR adaptation, comparatively few have looked at these simultaneously. The present study constitutes one such exploration: the effect of close-range fixation on VOR adaptation during head rotation.

In addition to primary rotational VOR system, the linear vestibulo-ocular (1-VOR) reflex system produces compensatory eye movements responding to signals from the otiliths and head acceleration. Since the eyes are offset from the axis of head rotation, translational motion of the eye occurs during all natural head movements (Crane \& Demer, 1997). The optimal mean VOR gain is above 1.0 (eye rotation/head rotation) for targets nearer than $6 \mathrm{~m}$, mainly determined by fixation distance (consistent with the inversesquare law), but also influenced by individual differences in the size of the head and inter-ocular distance. VOR gain is actively stabilized for all translational movement by both vestibular and extra-vestibular afferents to affect rapid compensation (Medendorp, Van Gisbergen, \& Gielen, 2002), with the additional non-vestibular components afforded by active head-rotation resulting in less retinal slip (Gielen, Gabal, \& Duysens, 2004).

In addition to gain amplification during near-target fixation, and gain attenuation when integrated with OKN, pursuit, and saccadic movements, open-loop VOR also adapts over time to systematic visual or vestibular alteration (this also occurs with 1-VOR; Seidman, Paige, \& Tomko, 1999). Both overall gain (Post \& Lott, 1993; Welch, Bridgeman, Williams, \& Semmler, 1998) and phase (Kramer, Shelhamer, Peng, \& Zee, 1998) changes can occur very rapidly when visual-vestibular relationships are disrupted. In natural conditions, both maturational size changes and damage to (or aging of) neural or muscular tissue require global adjustments in gain; in less natural conditions, altered optical or vestibular environments produce similar long-term effects. In the laboratory, these changes are often induced by pairing head movements with some alteration of the visual scene, using magnifying or minifying optical lenses (e.g., Collewijn, Martins, \& Steinman, 1983; Demer, Porter, Goldberg, Jenkins, \& Schmidt, 1989), or visual stimuli slaved to the head movements themselves (e.g., Post \& Lott, 1992; Welch et al., 1998).

Prolonged near-fixation may produce an environment where long-term VOR adaptation may occur: a non-modulated canal signal by itself is insufficient to produce complete compensation, requiring amplification by the distance signal. A comparison of this non-modulated signal to the oculomotor outcome (either eye position or retinal slip, see discussion) could result in increases of VOR gain that reduce the load on short-term compensatory systems. Alternatively, long-term VOR adaptation may not occur if: (1) the eye position signal employed for adaptation occurs after distance signal modulation; (2) retinal slip is necessary for adaptation; or (3) adaptation that would have otherwise 
occurred is actively suppressed since the system 'knows' that near fixations are occurring, perhaps it prevents adaptation utilizing some measure of near fixation (not necessarily the distance signal employed in short-term modulation) to cancel any changes in open-loop gain. The first step toward addressing these questions is to determine whether it is in fact possible for prolonged fixation of a near target to produce long-term gain adaptation. We attempted to adapt VOR through relatively demanding conditions: prolonged near-target fixation $(15 \mathrm{~cm})$ for six minutes, employing a single rotational frequency (and amplitude) to maximize adaptation (Lisberger, Miles, \& Optican, 1983).

\section{Method}

\subsection{Participants}

Three male and three female undergraduate psychology students ranging in age from 18 to 22 years participated in the experiment in order to fulfill requirements for undergraduate psychology courses. All but one had either normal or vision corrected with contact lenses only. One participant who was myopic participated with glasses removed, which may have slightly elevated measured gain in both baseline and post-adaptation phases for that individual. The experiment was conducted with approval of the University of California, Santa Cruz, Institutional Review Board in accordance with the 1964 Helsinki Declaration, including an informed consent process.

\subsection{Apparatus}

Participants sat in front of an opaque hemicylindrical screen (viewing distance $=90 \mathrm{~cm}$ ). There were two visual targets during the experiment: (1) a spot of red laser light reflected from a mirror onto the hemifield in front of them (both the laser itself and the mirror were shielded from view), and (2) a red LED attached to a moveable arm. Perceptually, both targets were of the same approximate size, and when overhead lights were extinguished, each target was the only visible object in the darkened room. Head position was measured using a Polhemus Fastrak magnetic field emitter unit in conjunction with a receiver mounted on a helmet tightly strapped to the participant's head.

Eye position was recorded (at $43 \mathrm{~Hz}$ ) by paired infrared sensitive photocells attached to the helmet and positioned below the participant's right eye. Error in the system was about $\pm 0.1^{\circ}$. The system was calibrated for each participant by having them look at targets to the left, center, and right of the centerline at the height of the adaptation targets, and equating the gains on the two sides. Custom software converted (online) the output of the eye monitor into degrees. Head and eye positions were visible to the experimenter throughout the experiment.

Passive whole-body rotation was accomplished via a sinusoidally rotating chair at $0.45 \mathrm{~Hz}, 30^{\circ}$ amplitude peak-to-peak.

\subsection{Design}

The protocol for each participant consisted of four phases:

(1) Measurement of baseline VOR - eye/head gain measured in darkness. 
(2) Adaptation phase - prolonged fixation upon the near target during passive wholebody rotation.

(3) Re-fixation on the distant stimulus - re-creating initial viewing distance.

(4) Re-measurement of VOR gain - eye/head gain measured in darkness.

\subsection{Instructions and preparation}

All instructions were given prior to the experiment, and then repeated as necessary during the trials to remind participants of their task(s). Participants were instructed not to make independent head movements throughout the experiment - a head rest on the back of the chair facilitated this requirement. They were also trained to not make saccades during the 5- and 10-s recording intervals, and practiced steady gaze before the experiment began.

Phase 1 (Measurement of baseline VOR): Participants were instructed to watch the laser spot ('far' target) when it was present, and to "keep looking where the spot was" when extinguished. Once chair oscillation was started, participants were shown the far target sec in an otherwise dark room for $15 \mathrm{~s}$. The target was then removed, and eye movements immediately measured in complete darkness for $5 \mathrm{~s}$. This sequence was repeated four times, resulting in four $5 \mathrm{~s}$ recordings of pre-exposure VOR gain.

Phase 2 (Adaptation phase): The chair was stopped for a period of $30 \mathrm{~s}$, during which the room was lit, and participants sat motionless, although allowed to make eye movements freely. This delay was logistically necessary as the experimenter had to extinguish the far target, and set up the near target on its adjustable arm. The latter was centrally placed $15 \mathrm{~cm}$ from the bridge of the nose of each participant. Lights were doused, passive chair-rotation re-initiated for $10 \mathrm{~s}$ in darkness, and the near-target turned on. Participants were instructed to watch this target until it was extinguished. The adaptation period lasted six minutes, and eye position was monitored to assure that participants were fixating the target (as evidenced by dramatic increases in the range of eye-position compared to that monitored in the far fixation condition).

Phase 3 (Re-fixation on the distant stimulus): Following the adaptation period, the chair was stopped, participants again sat motionless (except for saccades), and the overhead lights were turned on. To discharge any slow fusional or tonic vergence adaptation (Wolfe, Ciuffreda, \& Jacobs, 1987), another 30-s delay occurred, during which participants made numerous free saccades without head motion, as monitored by the experimenter. Also, the near fixation target was removed, and far target prepared. Participants were then asked to close their eyes for $10 \mathrm{~s}$ (to prevent re-adaptation during rotation) while the chair was aligned straight ahead. Lights were extinguished, and participants opened their eyes. With the chair still static, $10 \mathrm{~s}$ of darkness was followed by $10 \mathrm{~s}$ of exposure to the far target in order to center gaze straight ahead - participants were again instructed to "keep looking where the spot was" when it was extinguished.

Phase 4 (Re-measurement of VOR gain): After gaze was re-centered, chair rotation was re-established employing the same amplitude and frequency parameters as the adaptation period and pre-exposure measurements. Participants were again asked to look straight ahead at "where the laser spot used to be." After $10 \mathrm{~s}$ of rotation without measurement (to ensure sinusoidal chair rotation and allow time to remind participants to not make saccades), we began a $10 \mathrm{~s}$ recording period of post-exposure eye movements. 


\subsection{Measurement}

VOR gain was assessed by the ratio of mean velocity of eye movements to the mean velocity of head movements for each recording interval (easily measured as the rootmean-square amplitudes of the sinusoidal movements). The four gain measures of the pre-exposure recording intervals were averaged to arrive at an aggregate measure of pre-exposure gain for each participant. The single 10-s recording interval served as the post-exposure measure.

\section{Results}

Inspection of the eye movement records revealed a single small $\left(<0.5^{\circ}\right)$ saccade that occurred in each participant on every cycle of head rotation just after maximum eye acceleration when the VOR reversed direction. These 'catch-up' saccades occurred both preand post-exposure, and were identical to those discovered in previous research using the same apparatus (for a graphical example of eye and head records, see Fig. 1, in Welch et al., 1998). Gain changes produced by removing this small saccade were negligible, and disappeared when rounding to appropriate significant digits. Our instructions prior to each recording interval to maintain fixation were successful, as no other saccades were present in the eye movement record. No phase shifts were observed within the temporal parameters of our measurement capacity $(43 \mathrm{~Hz})$. Single vision of the target indicated accurate vergence during exposure. One individual (participant 2 in Fig. 1) who normally wore glasses participated with glasses removed, which may have slightly artificially elevated measured gain.

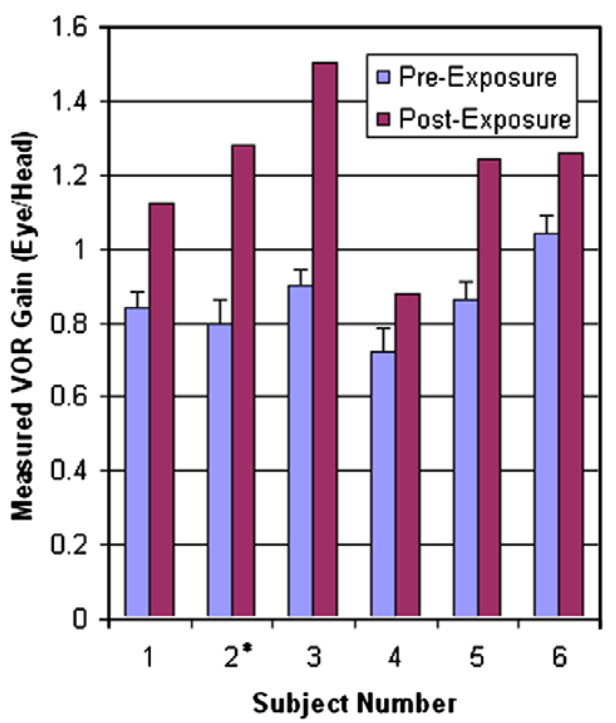

Fig. 1. Pre- and post-adaptation gains for all participants. Error bars for the baseline condition indicate standard deviation for the four recording intervals $(*$ indicates the myopic individual who participated with glasses removed). 
Pre-exposure VOR gain was in the expected range for passive whole-body rotation in the dark: $M=0.86, \mathrm{SE}=0.04$. Subsequent measurement for the post-adaptation period revealed large increases in measured VOR gain: $M=1.2, \mathrm{SE}=0.08$, which were statistically significant, $t(5)=5.2, p=.003$. Gain increased for each participant (see Fig. 1). If perfect near-focus compensation had been fully translated into global gain changes, we would have expected average gain to increase to 1.5 (eye/head). Since measured post-adaptation gain was 1.2, VOR compensation was $80 \%$ of full transfer (see Appendix for derivation of these equations). However, when one considers that baseline VOR rotation among participants was .86 (or $86 \%$ of optimal), virtually all the gain increases evoked by near-fixation resulted in global adaptation.

It is possible that if people improve in localizing the extinguished target in the final recording period, this could produce differences between pre- and post-adaptation measurements. However, to account for the current effects, such a change would necessitate a fixation beyond the $90 \mathrm{~cm}$ target distance (a closer fixation point would not increase the reported differences between pre- and post-, but rather reduce them). However, any differences between gain measured at $90 \mathrm{~cm}$ fixation, and those measured at above $90 \mathrm{~cm}$ fixation are very small. Thus any changes in VOR gain beyond a fixation of $90 \mathrm{~cm}$ are negligible, and any accentuation of reported effects minimal.

Given that the measured pre-adaptation gain is within normal parameters for passive rotation, and that post-adaptation gains are above 1.0, we do not believe this accounts for the effect.

\section{Discussion}

During near fixation, all necessary signals are present to adapt the VOR gain: head rotation speed, head translation speed, the distance signal, eye position and velocity, and possibly retinal slip (see below). In humans, vestibular canal signals modulate nearfixation VOR gain increases (particularly at high accelerations) at the very onset of head rotation, and thus distance scaling of VOR gain may occur in the classic three-neuron arc itself (Crane \& Demer, 1998). If true, a simple comparison employing the rotation-specific component would likely produce the long-term adaptation found under the current conditions. Clearly one possibility discussed in the introduction can be eliminated: the active cancellation of adaptation due to either the near-fixation distance signal or any other indicator of target distance. The current study finds that, akin to the interaction of pursuit and saccadic systems with short-term VOR modulation, the longer-term VOR adaptation system can be altered, reducing demand on the associated system (i.e., short-term distance modulation of VOR gain).

Originally, it was assumed that retinal slip was responsible for global adaptation in the VOR. However, Melville Jones, Berthoz, and Segal (1984) reported that global VOR gain attenuation can occur without any visual signal, by explicitly instructing human participants to 'imagine' a head fixed target during prolonged angular rotation. ${ }^{1}$ Consistent with

\footnotetext{
${ }^{1}$ The current adaptation protocol is quite different - participants are acutely aware of, and fixating, a stimulus that actually increases, not nulls the VOR gain required for compensation. Hine and Thorn (1987) found gain increases while participants imagined a near target, but subsequently measured gain while participants were still engaged in this task, i.e., at the near-fixation vergence angle.
} 
this finding are studies implicating a non-visual oculomotor signal in VOR gain adaptation for various non-primates (in cats, Ashton, Boddy, Dean, Milleret, \& Donaldson, 1988; in pigeons, Donaldson \& Knox, 1988; in rabbits, Collewijn \& Grootendorst, 1979). By inducing eye paralysis in rats, Gauthier, de'Speratti, Tempia, Marchetti, and Strata (1995) inferred that akin to signals employed in target localization (Bridgeman \& Stark, 1991), proprioception, not efference copy, signals VOR gain adaptation. Since relatively small amounts of retinal (foveal) slip occur during fixations at the distance we employed, adaptation under the current conditions are consistent with a comparison of pre-modulation canal and proprioceptive signals.

We employed a single frequency, velocity, and acceleration of head movement, as this has been shown to produce the most rapid adaptation when re-measured at those same values (Lisberger et al., 1983; Post \& Lott, 1993), as VOR adaptation is specific to the parameters of the rotation used to produce it (Wantanebe, Hattori, \& Koizuka, 2003). However, outside the laboratory, prolonged viewing of near stimuli would likely be accompanied by a range of head movements, and should generalize more widely. In fact, in these cases overall adaptation may be faster and/or more complete, since active head rotation has been shown to be more effective in producing adaptation than the passive rotation used in the present study (Collewijn et al., 1983).

The focal distance employed $(15.2 \mathrm{~cm}, 6 \mathrm{in}$.) is not uncommon for reading, particularly for near-sighted individuals, and even at the average reading distance of $16 \mathrm{in}$., gain increases are still significant (1.2, eye/head). Therefore, prolonged fixation could potentially disrupt performance when re-focusing to a far target. Lengthy viewing of such stimuli as a computer monitor would be likely to produce long-term VOR adaptation, a phenomenon likely to increase as the size of displays gets larger (encouraging more head movements). Under such circumstances, a person would likely experience incomplete VOR compensation upon viewing distant stimuli, including apparent concomitant motion when looking away from the near target (Tietz \& Gogel, 1978). While this may not have serious practical consequences in many environments, given the magnitude of VOR adaptation affected in the current study, in some cases the effects might be problematic. For example, prolonged attention to near instrumentation may be troublesome to aviators and others when fixation returns to distant targets. Designers of artificial visual environments should consider some of these unwanted effects when implementing such systems (see Draper, 1996 for a discussion of some of these issues).

One possible mitigation of deleterious effects may lie in the phenomena of dual adaptation - akin to dysmetria produced with prisms (e.g., Welch, Bridgeman, Anand, \& Browman, 1993). Repeated exposures to altered visuo-vestibular environments that demand different VOR gains may result in faster adaptation rates to both environments over time. Often some differentiating cue (e.g., orientation, Baker, Perlmutter, Peterson, Rude, \& Robinson, 1987; Yakushin, Raphan, \& Cohen, 2000; eye position, Shelhamer, Robinson, \& Tan, 1992) precedes these changes, but need not - the mismatched environment itself can serve as the indicator of a necessary gain change (Welch et al., 1998). We suspect that context-dependent adaptation would occur for near-target driven VOR change, but ultimately the question is empirical and merits further investigation. There exist some discrepancies regarding dual adaptation of VOR in the literature. One possible explanation may be that adaptation is heterogeneous: that affected by point light sources (i.e., foveal) may differ from that produced by whole-field movement (Shelhamer \& Zee, 2003). If slip/OKN-driven adaptation is qualitatively different from proprioceptive (or 
pursuit) driven adaptation, the implications would be important in a number of applied settings, and would also affect the interpretation of the current results.

\section{Acknowledgement}

The present research was supported by a Cooperative Agreement between the University of California, Santa Cruz, and the National Aeronautics and Space Administration (Project UPN 199-16-12-34). The authors thank Clifton Schor of the School of Optometry, University of California, Berkeley, for the use of the rotating chair.

\section{References}

Ashton, J. A., Boddy, A., Dean, S. R., Milleret, C., \& Donaldson, I. M. (1988). Afferent signals from the extraocular muscles in the medial vestibular nucleus, the nucleus prepositus hypoglossi and adjacent brainstem structures. Neuroscience, 26, 131-145.

Baker, J. F., Perlmutter, B. W., Peterson, S. A., Rude, S. A., \& Robinson, F. R. (1987). Simultaneous opposing adaptive changes in cat vestibulo-ocular reflex direction for two body orientations. Experimental Brain Research, 69, 220-224.

Bridgeman, B., \& Stark, L. (1991). Ocular proprioception and efference copy in registering visual direction. Vision Research, 31, 1903-1913.

Collewijn, H., \& Grootendorst, A. F. (1979). Adaptation of optokinetic and vestibule-ocular reflexes to modified visual input in the rabbit. In R. Granit \& O. Pompeiano (Eds.), Reflex control of posture and movement (pp. 771-781). Amsterdam: Elsevier.

Collewijn, H., Martins, A. J., \& Steinman, R. M. (1983). Compensatory eye movements during active and passive head movements: Fast adaptation to changes in visual magnification. Journal of Physiology, 340, 259-286.

Crane, B. T., \& Demer, J. L. (1997). Human gaze stabilization during natural activities: Translation motion magnification and target distance effects. Journal of Neurophysiology, 78, 2129-2144.

Crane, B. T., \& Demer, J. L. (1998). Human horizontal vestibule-ocular reflex initiation: Effects of acceleration, target distance, and unilateral deafferentation. Journal of Neurophysiology, 80, 1151-1166.

Demer, J. L., Porter, F. I., Goldberg, J., Jenkins, H. A., \& Schmidt, K. (1989). Adaptation to telescopic spectacles: Vestibule-ocular reflex plasticity. Investigative Opthalmology and Vision Science, 30, 159-170.

Donaldson, I. M., \& Knox, P. C. (1988). Units in pigeon brainstem whose vestibular responses are modulated by passive eye movement. Journal of Physiology (London), 398, 35.

Draper, M. H. (1996). Can your eyes make you sick? Investigating the relationship between the vestibule-ocular reflex and virtual reality (retrieved 02.05.06). http://www.hitl.washington.edu/publications/r-96-3/.

Gauthier, G. M., de'Speratti, C., Tempia, F., Marchetti, E., \& Strata, P. (1995). Influence of eye motion on adaptive modifications of the vestibule-ocular reflex in the rat. Experimental Brain Research, 103, 393-401.

Gielen, C. C., Gabal, S. F., \& Duysens, J. (2004). Retinal slip during active head motion and stimulus motion. Experimental Brain Research, 155, 211-219.

Grossman, G. E., Leigh, R. J., Bruce, E. N., Huebner, W. P., \& Lanska, D. J. (1989). Performance of the human vestibuloocular reflex during locomotion. Journal of Neurophysiology, 62, 264-272.

Guitton, D., \& Volle, M. (1987). Gaze control in humans: Eye-head coordination during orienting movements to targets within and beyond the oculomotor range. Journal of Neurophysiology, 58, 427-459.

Hine, T., \& Thorn, F. (1987). Compensatory eye movements during active head rotation for near targets: Effects of imagination, rapid head oscillation and vergence. Vision Research, 27, 1639-1657.

Kramer, P. D., Shelhamer, M., Peng, G., \& Zee, D. S. (1998). Context-specific short-term adaptation of the phase of the vestibule-ocular reflex. Experimental Brain Research, 120, 184-192.

Lisberger, S. G. (1990). Visual tracking in monkeys: Evidence for short-latency suppression of the vestibuloocular reflex. Journal of Neurophysiology, 63, 676-688.

Lisberger, S. G., Miles, F. A., \& Optican, L. M. (1983). Frequency-selective adaptation: Evidence for channels in the vestibule-ocular reflex? The Journal of Neuroscience, 3, 1234-1244.

Medendorp, Q. P., Van Gisbergen, J. A. M., \& Gielen, C. C. A. M. (2002). Human gaze stabilization during active head translation. Journal of Neurophysiology, 87, 295-304. 
Melville Jones, G., Berthoz, A., \& Segal, B. (1984). Adaptive modification to the vestibulo-ocular reflex by mental effort in darkness. Experimental Brain Research, 56, 149-153.

Post, R. B., \& Lott, L. A. (1992). The relationship between vestibulo-ocular reflex plasticity and changes in apparent concomitant motion. Vision Research, 32, 89-96.

Post, R. B., \& Lott, L. A. (1993). Frequency specificity in the adaptation of apparent concomitant motion. Bulletin of the Psychonomic Society, 31, 53-56.

Seidman, S. H., Paige, G. D., \& Tomko, D. L. (1999). Adaptive plasticity in the naso-occipital linear vestibuleocular reflex. Experimental Brain Research, 125, 485-494.

Shelhamer, M., Robinson, D. A., \& Tan, S. (1992). Context-specific adaptation of the gain of the vestibulo-ocular reflex in humans. Journal of Vestibular Research, 2, 89-96.

Shelhamer, M., \& Zee, D. S. (2003). Context-specific adaptation and its significance for neurovestibular problems of space flight. Journal of Vestibular Research, 13, 345-362.

Tietz, J. D., \& Gogel, W. C. (1978). Adaptation to apparent concomitant motion in the absence of physical or retinal motion. Bulletin of the Psychonomic Society, 12, 1-4.

Wantanebe, S., Hattori, K., \& Koizuka, I. (2003). Flexibility of vestibulo-ocular reflex adaptation to modified visual input in human. Auris Nasus Larynx, 30, S29-S34.

Welch, R. B., Bridgeman, B., Anand, S., \& Browman, K. E. (1993). Alternating prism exposure causes dual adaptation and generalization to a novel displacement. Perception and Psychophysics, 54, 195-204.

Welch, R. B., Bridgeman, B., Williams, J. A., \& Semmler, R. (1998). Dual adaptation and adaptive generalization of the human vestibulo-ocular reflex. Perception and Psychophysics, 60, 1415-1425.

Wolfe, K. S., Ciuffreda, K. J., \& Jacobs, S. E. (1987). Time course and decay of effects of near work on tonic accommodation and tonic vergence. Opthalmology and Physiological Optics, 7, 131-135.

Yakushin, S. B., Raphan, T., \& Cohen, B. (2000). Context-specific adaptation of the vertical vestibuloocular reflex with regard to gravity. Journal of Neurophysiology, 84, 3067-3071. 